\title{
Development and evaluation of an in-house ELISA to detect hepatitis B virus surface antigen in resource-limited settings
}

\author{
Fatema $\mathrm{K}^{1}$, Tabassum $\mathrm{S}^{2}$, Nessa $\mathrm{A}^{2}$, Jahan $\mathrm{M}^{2}$ \\ ${ }^{l}$ Department of Pathology \& Microbiology, City Dental College, Malibagh Chowdhury Para, Dhaka, \\ ${ }^{2}$ Department of Virology, Bangabandhu Sheikh Mujib Medical University, Shahbag, Dhaka. \\ Email: fatemadr.kaniz@yahoo.com
}

\begin{abstract}
Hepatitis B virus (HBV) infection is of global public health concern. Among various serological tests used for the diagnosis and screening of HBV infection, the enzyme-linked immunosorbent assay (ELISA) to detect hepatitis B surface antigen ( $\mathrm{HbsAg}$ ) is most widely used. The present study was designed to develop and standardize a cost effective in-house ELISA for the detection of HbsAg and compare its performance with two established commercial kits. The concentrations of coating antibody, conjugates and sera were fixed by checkerboard titration. Using known HBsAg positive and negative sera, four different concentrations $(1,0.5,0.25$ and $0.125 \mu \mathrm{g} /$ well) of coating anti-HBs were applied. Similarly, serial dilutions of patients' sera (1 in 2, 1 in 3, 1 in 5 and 1 in 9) and conjugates (1 in 2,1 in 3,1 in 5,1 in 9 and 1 in 17) were evaluated by checkerboard titration. The optimal concentration of coating antibody was determined at $0.25 \mu \mathrm{g} /$ well and 1 in 9 dilution for both conjugates and sera. The performance comparison of our in-house ELISA showed excellent correlation with two commercial kits (Pearson 0.957, $\mathrm{P}=0.001$ for monoclonal antibody coated kit and Pearson $0.929, \mathrm{P}=0.000$ for polyclonal antibody coated kit) when $\mathrm{OD}$ values were compared. All commercial kit proven positive samples was positive while all negative samples were negative with the in-house ELISA resulting in $100 \%$ sensitivity and specificity. The results of our study demonstrated that our inhouse ELISA for detection of HBsAg was equally as sensitive and specific as two well-known commercial kits. Thus, this system may be a useful tool for diagnostic and screening purposes, as well as outbreak investigations.
\end{abstract}

\section{Introduction}

Hepatitis B virus (HBV) infection represents a global public health problem mainly affecting developing countries. Worldwide, about 2 billion people are infected with $\mathrm{HBV}$, resulting in a million deaths ${ }^{\mathbf{1 , 2}}$. Over 350 million people become chronically infected, of whom approximately three quarters are in Asia and the western pacific region $^{3,4}$. HBV is rapidly spreading in developing countries including Bangladesh which is categorized as an intermediate endemic zone for HBV with prevalence rates of 0.7 to $7.5 \%$ among different populations ${ }^{5,6}$. Various serological markers like $\mathrm{HBsAg}$, anti-HBs, $\mathrm{HBeAg}$ and anti$\mathrm{HBc}$ are assessed to determine the infection status of $\mathrm{HBV}^{\mathbf{7 , 8}}$. Among these, the hepatitis $\mathrm{B}$ surface antigen (HBsAg) is the most widely used marker for diagnosis and screening of HBV infection. An individual positive for $\mathrm{HBs} \mathrm{Ag}$ is considered to be infected with $\mathrm{HBV}$, and is therefore potentially infectious?. HBsAg is also a significant tool for predicting the outcome of HBV infection ${ }^{\mathbf{1 0}}$.
Detection of HBsAg is usually performed by enzyme immunoassays (EIA)s, which can detect HBsAg at concentrations of $\geq 0.1 \mathrm{ng} / \mathrm{ml}^{11}$. Moreover, the ease of availability and high sensitivity of ELISA makes this method more affordable and applicable in comparison to expensive and labor-intensive assays ${ }^{12}$. The cost of imported ELISA kits is usually quite high and not affordable by the average diagnostic laboratories in resource-limited countries. As such, the present study describes the development and evaluation of a simple and cost-effective in-house ELISA for the determination of $\mathrm{HbsAg}$ which is suitable for resource-poor settings.

\section{Materials and Methods}

This experimental study was carried out at the Department of Virology, Bangabandhu Sheikh Mujib Medical University (BSMMU), Shahbag, Dhaka from July 2009 to June 2010.

Polyclonal antibody: Polyclonal antibody to HBsAg (anti-Hbs) was commercially obtained 
(Meridian Life Science, USA). It contained $1 \mathrm{mg}$ antibody in $1 \mathrm{ml}$.

Human sera: Human sera used for this study were collected from residual sera sent to the Diagnostic Virology laboratory of BSMMU for diagnostic purposes. Each serum was tested for HBsAg by monoclonal antibody coated commercial ELISA plates and classified as HBsAg positive or negative. After development of in-house ELISA to evaluate its performance, monoclonal antibody coated commercial kit verified human sera were used. By non probability convenience sampling, 90 samples were collected, of which, 42 samples were HBsAg positive and 48 samples were $\mathrm{HBsAg}$ negative. These 90 verified serum samples were then tested with the in-house ELISA and with two commercial kits; a polyclonal antibody coated and a monoclonal antibody coated kit to detect and compare their optical density (OD) values.

The test procedure of in-house ELISA was as follows: Briefly, anti-HBs was diluted in carbonatebicarbonate buffer $(\mathrm{pH}$ 9.6) with concentration ranging from 1.25 to $10 \mu \mathrm{g} / \mathrm{ml}(0.125$ to $1 \mu \mathrm{g} /$ well). Then, ELISA micro titer plates were coated with anti-HBs diluted in coating buffer $(100 \mu 1 /$ well). The plate was coated vertically using columns 1-4. Four different antibody coating concentrations (10, $5,2.5$ and $1.25 \mu \mathrm{g} / \mathrm{ml}$ ) were tested with each concentration added to one vertical column. Plate was incubated overnight at $4{ }^{\circ} \mathrm{C}$, and unbound sites were blocked with $1 \%$ bovine serum albuminphosphate buffer solution (BSA-PBS) and incubated again for 3 hours at $37^{\circ} \mathrm{C}$. After 5 washes with PBS Tween 20 (PBS-T) and drying, $25 \mu \mathrm{l} /$ well of conjugate 1 (anti-HBs/Biotin) was added to 100 $\mu 1$ of undiluted samples, incubated at $37^{\circ} \mathrm{C}$ for 1 hour, and washed 5 times with PBS-T. Then, 100 $\mu 1 /$ well of conjugate 2 (Steptovidin/peroxidase) was dispensed, plates were sealed and incubated at $37^{\circ} \mathrm{C}$ for $30 \mathrm{~min}$, washed 5 times with PBS-T and dried.

Each well was then filled with $75 \mu$ of the working chromogen solution $[1 \mathrm{ml}$ of substrate chromogen with $10 \mathrm{ml}$ of substrate Tetramethylbenzidine (TMB)], sealed and incubated in the dark at 15 $25^{\circ} \mathrm{C}$ for $30 \mathrm{~min}$. Reaction was stopped by adding $75 \mu \mathrm{l} /$ well of stopping solution $\left(1 \mathrm{M} \mathrm{H}_{2} \mathrm{SO}_{4}\right.$ ). Finally, the optical density (OD) was measured at $450 \mathrm{~nm}$ wavelength with an ELISA plate reader (Organotechnica, Austria, Model 530). After fixing the concentration of anti-HBs, the same procedure was repeated with other concentrations of samples i.e. 1 in 2,1 in 3 and 1 in $5 \& 1$ in 9 dilutions and conjugates i.e. 1 in 2, 1 in 3 and 1 in 5, 1 in 9 and 1 in 17 dilutions.
Data Analysis: OD values of the in-house ELISA were analyzed for sensitivity and specificity \& also compared with OD values of the two commercial assays by Pearson correlation coefficient. SPSS version 16 was used for statistical calculations.

\section{Results}

Table I shows the optimal concentrations of sera and reagents determined for in-house ELISA in the present study. The final concentration of coating anti-HBs was fixed at $0.25 \mu \mathrm{g} / \mathrm{well}$, while that for sera and conjugates were fixed at 1 in 9 dilution.

ELISA Validation: The OD values of the HBsAg positive and HBsAg negative samples measured with in-house ELISA are shown in Figure $1 \& 2$ respectively. Two ELISA plates were used. In plate 1, 1-24 positive and 1-40 negative samples and in plate 2, 25-42 positive and 41-48 negative samples were tested. Cut-off values in plate 1 and 2 were 0.123 and 0.133 respectively. Fig 1 shows that OD values of all the positive samples were above the cut-off value and Fig 2 shows that OD values of all negative samples were below the cut-off value.

Comparison Studies: All positive and negative samples were again tested using commercially available polyclonal and monoclonal $\mathrm{HbsAg}$ ELISA kits. Samples were used in undiluted form. Same serial numbers of the samples as in the case of in-house ELISA were maintained. Fig 3 shows the correlation of OD values obtained by in-house ELISA and polyclonal antibody coated commercial kit. A high correlation coefficient (Pearson 0.929, $\mathrm{P}<0.000)$ was observed when OD values were compared. Fig 4 shows comparison of OD values obtained by in-house ELISA and monoclonal antibody coated commercial ELISA plate. A high correlation coefficient (Pearson 0.957, $\mathrm{P}<0.001$ ) was also observed when OD values were compared.

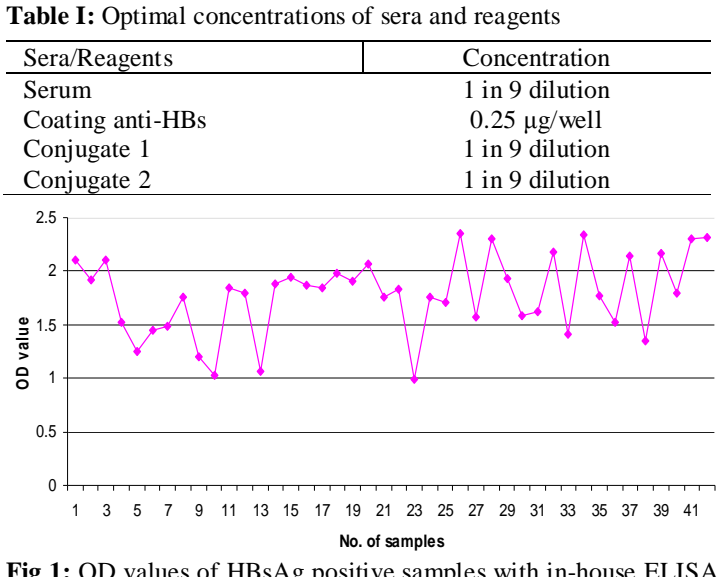

Fig 1: OD values of HBsAg positive samples with in-house ELISA (Cut-off value for samples $1-24$ is 0.123 and for samples 25-42 is $0.133)$ 


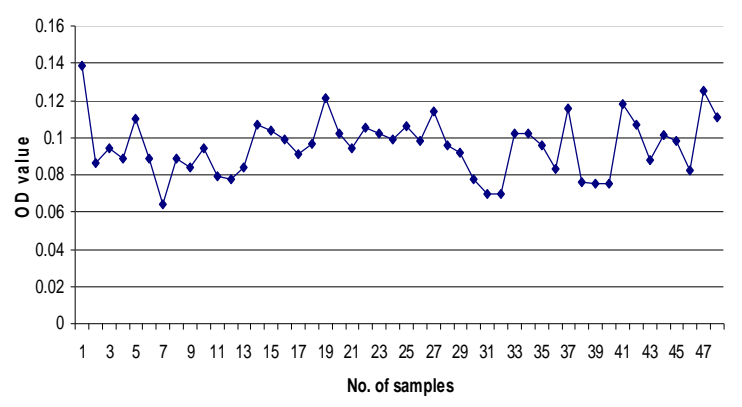

Fig 2: OD values of HBsAg negative samples with in-house ELISA (Cut-off value for samples $1-40$ is 0.123 and for samples $41-48$ is 0.133 )

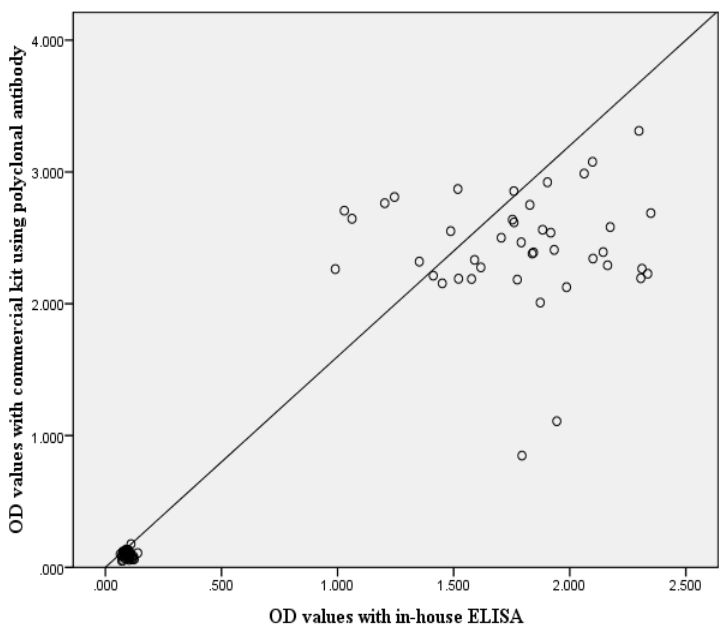

Fig 3: Correlation of OD values obtained by in-house ELISA using polyclonal antibody and commercial kit using polyclonal antibody.

Upper scattered circles $=$ OD values of positive samples Lower clustered circles $=$ OD values of negative samples

Each circle represents corresponding OD values of a sample obtained by in-house ELISA using polyclonal antibody and commercial kit using polyclonal antibody.

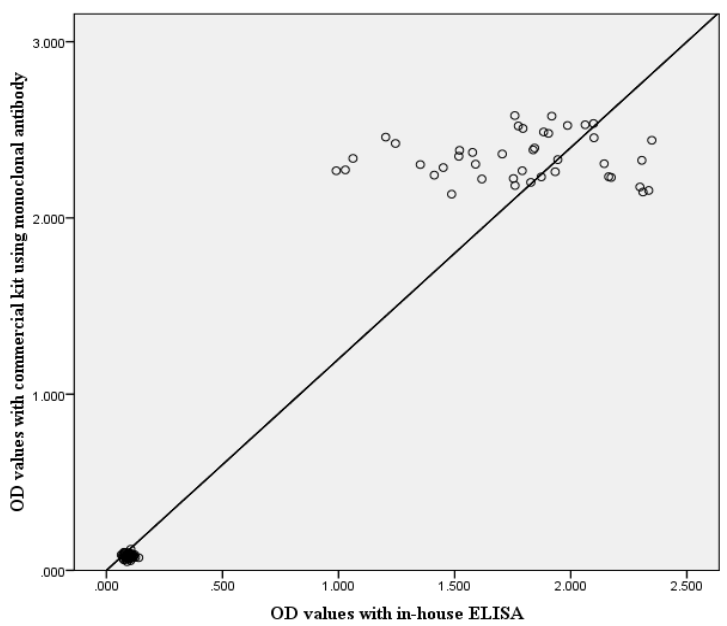

Fig 4: Correlation of OD values obtained by in-house ELISA using polyclonal antibody and commercial kit using monoclonal antibody. Each circle represents corresponding OD values of a sample obtained by in-house ELISA using polyclonal antibody and commercial kit using monoclonal antibody.

\section{Discussion}

Hepatitis B surface antigen (HBsAg) has been the principal target for laboratory testing to identify active infection by HBV. Among the many commercially licensed HBsAg assays available, enzyme-linked immunosorbent assays (ELISA)s are currently the most frequently used as they are most appropriate for screening large number of specimens ${ }^{13}$. Moreover, it is also useful for surveillance studies which provide critical information for guiding public health decisions related to this disease. During these surveillance studies, participating laboratories receive large numbers of samples that are tested in batches. Although rapid tests are more suitable and convenient than ELISA for bedside testing, inadequately equipped or field laboratories, and in small-scale, an effective ELISA is superior in routine surveillance studies or outbreak investigations where high throughput is important.

Despite its high-level performance, there is a continuous challenge regarding the sensitivities of various HBsAg detection systems so that the residual risk of transfusion associated $\mathrm{HBV}$ infection can be minimized and the window period can be further reduced ${ }^{\mathbf{1 4}}$. Using more sensitive assay systems, low levels of $\mathrm{HBsAg}$ can be detected so that the residual risk of transfusion associated HBV infection can be minimized ${ }^{15}$. By using in-house ELISA, sensitivity and specificity can be established for a particular assay. Moreover, it is also cost-effective to run in-house ELISAs ${ }^{16,17}$. While commercially available ELISA kits are used for HBsAg detection at different diagnostic laboratories, they are quite expensive for developing countries as these kits are imported from abroad. In addition to its price, it also includes transport expenditure and taxes, thereby increasing the total cost. Furthermore, the procedure of importing is cumbersome and time consuming. These problems can be overcome and valuable foreign currency can be saved by developing an inhouse ELISA for HBsAg locally.

In response to this need, we developed an in-house ELISA for the detection of HbsAg. The performance of this in-house ELISA was evaluated against two well-established commercial ELISA kits. Firstly, to compare the performance of the inhouse ELISA, commercial kit using monoclonal antibody verified $\mathrm{HBsAg}$ positive and negative samples were used and the results were compared. The same samples were also compared with commercial kits using polyclonal antibody. All commercial kit proven positive samples were positive, whereas all negative samples were negative with the in-house ELISA, whereby both 
the sensitivity and specificity of our in-house ELISA was $100 \%$. However, with commercial kits, undiluted (neat) patients' sera were used, but for our in-house ELISA, 1 in 9 dilution sera gave optimal results. Therefore, our in-house ELISA could detect HBsAg at lower concentrations and had a better sensitivity in comparison to commercial kits. Furthermore, using the cut off value obtained from HBsAg negative samples, both sensitivity and specificity were maximised to $100 \%$.

The OD values obtained from our in-house ELISA were compared with OD values obtained from commercial kits using both polyclonal and monoclonal antibodies. In-house ELISA reported in our study showed excellent correlation with both commercial kits when OD values of in-house ELISA were compared (Pearson correlation coefficient $0.929, \mathrm{P}<0.0001$ with commercial kit using polyclonal antibody and Pearson correlation coefficient was $0.957, \mathrm{P}<0.001$ with commercial kit using monoclonal antibody). Antigen concentration was not measured by our in-house ELISA because standard HBsAg with known concentration was not used in this study due to various constraints as compared to other studies ${ }^{\mathbf{1 2 , 1 8}}$. Therefore, the lowest antigen concentration could not be determined.

Conclusion: The results of our in-house ELISA reflected a reliable method for the detection of HBsAg. It had sensitivity and specificity comparable to commercial assay for $\mathrm{HBsAg}$ detection. The in-house ELISA could detect antigen from serum at a concentration 9 times lower than that used in commercial kits and was also cost effective. Thus, the in-house ELISA may be adopted for screen large number of samples in resource-constrained settings like Bangladesh. However, the preliminary results obtained from our study require further confirmation by repeated testing and evaluation in different settings including rural areas.

\section{Acknowledgement}

The authors gratefully acknowledge the expert technical assistance of Dr. Rashedul Haque, Scientist and Head, Parasitology Laboratory, ICDDR,B.

\section{References}

1. Lavanchy D. Hepatitis B virus epidemiology, disease burden, treatment, and current and emerging prevention and control measures. J Viral Hepat 2004; 11: 97-107.

2. Kermode M. Unsafe injections in low-income country health settings: need for injection safety promotion to prevent the spread of blood-borne viruses. Health Promot Int 2004; 19: 95-103.

3. Sherlock S, Dooley J. Diseases of the Liver and Biliary System. 11th ed. Blackwell publishing, 2002: 285-303.

4. Chub-uppakarn S, Panichart P, Theamboonlers A, Poovarawan Y. Impact of the hepatitis B mass vaccination program in the southern part of Thailand. Southeast Asian J Trop Med Public Health 1998; 29: 464-8.

5. Edmunds WJ, Medley GF, Nokes DJ, Hall AJ, Whittle HC. The influence of age on the development of the Hepatitis B carrier state. Proc R Soc Lond B Biol Sci 1993; 253: 197-201.

6. Mohanty SR, Kupfer SS, Khiani V. Treatment of chronic hepatitis B. Nat Clin Pract Gastroenterol Hepatol 2006; 3: 446-58.

7. Hoofnagle JH. Serologic markers of hepatitis B virus infection. Annu Rev Med 1981; 32: 1-11.

8. Kao JH. Diagnosis of hepatitis B virus infection through serological and virological markers. Expert Rev Gastroenterol Hepatol 2008; 2(4): 553-562.

9. World Health Organization. Hepatitis B surface antigen assays: operational characteristics (Phase I) Report 1. Blood safety and Clinical Technology, WHO, 2001; Geneva.

10. Joller-Jemelka HI, Pfister HF, Grob PJ. The prognostic significance of quantitative $\mathrm{HBsAg}$ determination in acute hepatitis B. Schweiz Med Wochenschr 1985; 115 (37): 1249-1256.

11. Mahoney FJ. Update on diagnosis, management, and prevention of hepatitis B virus infection. Clin Microbiol Rev 1999; 12(2): 351-366.

12. Karakus R, Aral LA, Basturk B, Aybay C. Development of a highly sensitive ELISA for quantification of hepatitis B virus (HBV) surface antigen (HBsAg). Turk J Med Sci 2007; 37(2): 87-92.

13. World Health Organization. Hepatitis B surface antigen assays: operational characteristics (Phase I) Report 2. Department of Essential Health technologies, WHO, 2004; Geneva.

14. Candotti D, Allain JP. Transfusion-transmitted hepatitis B virus infection. J Hepatol 2009; 51(4): 798-809.

15. Hatzakis A, Magiorkinis E, Haida C. HBV virological assessment. J Hepatol 2006; 44: S71-S76.

16. Fadeel MA, House BL, Wasfy MM, et al. Evaluation of a newly developed ELISA against Widal, TUBEXTF and Typhidot for typhoid fever surveillance. J Infect Dev Ctries 2011; 5(3): 169-175.

17. Aybay C, Karakus R, Gundogdu AG. Development of a diagnostic and screening ELISA system for measuring Tetanus antitoxoid levels. Turk J Med Sci 2003; 33: 289-294.

18. Liu V, Green A. A monoclonal-antibody enzyme immunoassay for detection of hepatitis B surface antigen with use of a biotin-avidin system. Clin Chem 1985; 31(2): 202-205. 